\title{
Stability of Phase Retrievable Frames
}

\author{
Radu Balan \\ Mathematics Department and CSCAMM, University of Maryland, \\ College Park, MD 20742, USA
}

July 27,2018

\begin{abstract}
In this paper we study the property of phase retrievability by redundant sysems of vectors under perturbations of the frame set. Specifically we show that if a set $\mathcal{F}$ of $m$ vectors in the complex Hilbert space of dimension $\mathrm{n}$ allows for vector reconstruction from magnitudes of its coefficients, then there is a perturbation bound $\rho$ so that any frame set within $\rho$ from $\mathcal{F}$ has the same property. In particular this proves the recent construction in [15] is stable under perturbations. By the same token we reduce the critical cardinality conjectured in [1] to proving a stability result for non phase-retrievable frames.
\end{abstract}

\section{INTRODUCTION}

The phase retrieval problem presents itself in many applications is physics and engineering. Recent papers on this topic (see [8, 18, 6, 7, 1, 11, 51]) present a full list of examples ranging from X-Ray crystallography to audio and image signal processing, classification with deep networks, quantum information theory, and fiber optics data transmission.

In this paper we consider the complex case, namely the Hilbert space $H=\mathbb{C}^{n}$ endowed with the usual Euclidian scalar product $\langle x, y\rangle=\sum_{k=1}^{n} x_{k} \overline{y_{k}}$. On $H$ we consider the equivalence relation $\sim$ between two vectors $x, y \in H$ defined as follows; the vectors $x$ and $y$ are similar $x \sim y$ if and only if there is a complex constant $z$ of unit magnitude, $|z|=1$, so that $y=z x$. Let $\hat{H}=H / \sim$ be the quotient space. Thus an equivalence class (a ray) has the form $\hat{x}=\left\{e^{i \varphi} x, \varphi \in[0,2 \pi)\right\}$. A subset $\mathcal{F} \subset H$ of the Hilbert space $H$ (regardless whether it is finite dimensional or not) is called frame if there are two positive constants $0<A \leq B<\infty$ (called frame bounds) so that for any vector $x \in H$,

$$
A\|x\|^{2} \leq \sum_{f \in \mathcal{F}}|\langle x, f\rangle|^{2} \leq B\|x\|^{2}
$$

In the finite dimensional case considered in this paper, the frame condition simply reduces to the spanning condition. Specifically $\mathcal{F}=\left\{f_{1}, \ldots, f_{m}\right\}$ is frame for $H$ if and only if $H=\operatorname{span}(\mathcal{F})$. Obviously $m \geq n$ must hold. When we can choose $A=B$ the frame is called tight. If furthermore $A=B=1$ then $\mathcal{F}$ is said a Parseval frame. Consider the following nonlinear map

$$
\alpha: \hat{H} \rightarrow \mathbb{R}^{m} \quad, \quad(\alpha(\hat{x}))_{k}=\left|\left\langle x, f_{k}\right\rangle\right|, 1 \leq k \leq m
$$

which is well defined on the classes $\hat{x}$ since $\left|\left\langle x, f_{k}\right\rangle\right|=\left|\left\langle y, f_{k}\right\rangle\right|$ when $x \sim y$. 
The frame $\mathcal{F}=\left\{f_{1}, \ldots, f_{m}\right\}$ is called phase retrievable is the nonlinear map $\alpha$ is injective. Notice that any signal $x \in H$ is uniquely defined by the magnitudes of its frame coefficients $\alpha(x)$ up to a global phase factor, if and only if $\mathcal{F}$ is phase retrievable. The main result of this paper states that the phase retrievable property is stable under small perturbations of the frame set. Specifically we show

Theorem 1.1 Assume $\mathcal{F}=\left\{f_{1}, \ldots, f_{m}\right\}$ is a phase retrievable frame for a complex Hilbert space $H$. Then there is a $\rho>0$ so that any set $\mathcal{F}^{\prime}=\left\{f_{1}^{\prime}, \ldots, f_{m}^{\prime}\right\}$ with $\left\|f_{k}-f_{k}^{\prime}\right\|<\rho, 1 \leq k \leq m$, is also a phase retrievable frame.

We prove this theorem in section 3 . The proof is based on a recent necessary and sufficent condition obtained independently in [11] and [7. The exact form of this result is slightly different than the equivalent results stated in the aforementioned papers. Consequently we will provide a direct proof.

An interesting problem on phase retrievable frames is to find a critical cardinal $m^{*}(n)$ that has the following properties:

(A) For any $m \geq m^{*}(n)$ the set of phase retrievable frames is generic with respect to the Zariski topology;

(B) If $\mathcal{F}$ is a phase retrievable frame of $m$ vectors, then $m \geq m^{*}(n)$.

Clearly (B) is equivalent to:

(C) If $m<m^{*}(n)$ there is no frame $\mathcal{F}$ of $m$ vectors that is also phase retrievable.

The current state-of-the-art on this problem is summarized by the following statements:

(i) see [[8]]. If $m \geq 4 n-2$ then generically (with respect to the Zariski topology) any frame is phase retrievable for $\mathbb{C}^{n}$;

(ii) see [44]]. For generic $4 n \times n$ unitary matrices on $\mathbb{C}^{n}$, any subset of $m=4 n-3$ columns forms a phase retrievable frame;

(iii) see [33]]. If $\mathcal{F}$ is a phase retrievable frame in $\mathbb{C}^{n}$ then

$$
m \geq 4 n-2-2 \beta+ \begin{cases}2 & \text { if } n \text { odd and } \beta=3 \bmod 4 \\ 1 & \text { if } n \text { odd and } \beta=2 \bmod 4 \\ 0 & \text { otherwise }\end{cases}
$$

where $\beta=\beta(n)$ is the number of 1's in the binary expansion of $n-1$.

Hence, if such a critical cardinal exists, we know $4 n-O(\log (n)) \leq m_{\mathbb{C}}^{*}(n) \leq 4 n-2$. The authors of [1] conjectured that $m_{\mathbb{C}}^{*}(n)=4 n-4$. In the case $m=4 n-4$, Bodmann and Hammen constructed [15] a phase retrievable frame. In section 4 we review their construction and we show it is stable under small perturbations. In section 5 we consider the critical cardinality conjecture and show that $(\mathrm{C})$ is equivalent to a stability result for sets of frames that fail to be phase retrievable.

Note the corresponding problems for the real case are completely solved. In fact 8 gives a geometric condition equivalent to a frame being phase retrievable in $\mathbb{R}^{n}$. That condition (namely, for any partition of the frame set $\mathcal{F}=\mathcal{F}_{1} \cup \mathcal{F}_{2}$, at least one of $\mathcal{F}_{1}$ or $\mathcal{F}_{2}$ must span $\mathbb{R}^{n}$ ) is stable under small perturbations. Additionally that same condition implies that $m_{\mathbb{R}}^{*}=2 n-1$ is the critical cardinal in the real case. 


\section{Notations}

In this section we recall some notations we introduced in [7] that will be used in the following sections. Let $\mathcal{F}=\left\{f_{1}, \ldots, f_{m}\right\}$ be a frame in $H=\mathbb{C}^{n}$. Let $\mathbf{j}: H \rightarrow \mathbb{R}^{2 n}$ denote the embedding

$$
\mathbf{j}(x)=\left[\begin{array}{c}
\operatorname{real}(x) \\
\operatorname{imag}(x)
\end{array}\right]
$$

which is a unitary isomorphism $\mathbf{j}$ between $H$ seen as a real vector space endowed with the real inner product $\langle x, y\rangle_{\mathbb{R}}=\operatorname{real}(\langle x, y\rangle)$ and $\mathbb{R}^{2 n}$ :

$$
\langle x, y\rangle_{\mathbb{R}}=\operatorname{real}(\langle x, y\rangle)=\langle\mathbf{j}(x), \mathbf{j}(y)\rangle .
$$

For two vectors $u, v \in \mathbb{R}^{2 n}, \llbracket u, v \rrbracket$ denotes the symmetric outer poduct

$$
\llbracket u, v \rrbracket=\frac{1}{2}\left(u v^{T}+v u^{T}\right) .
$$

and similarly for two vector $x, y \in \mathbb{C}^{n}$ denote by $\llbracket x, y \rrbracket$ their symmetric outer product defined by

$$
\llbracket x, y \rrbracket=\frac{1}{2}\left(x y^{*}+y x^{*}\right)
$$

For each $n$-vector $f_{k}$ we denote by $\varphi_{k}$ the $2 n$ real vector, and by $\Phi_{k}$ the symmetric nonnegative rank- 2 , $2 n \times 2 n$ matrix defined by

$\varphi_{k}=\mathbf{j}\left(f_{k}\right)=\left[\begin{array}{c}\operatorname{real}\left(f_{k}\right) \\ \operatorname{imag}\left(f_{k}\right)\end{array}\right], \Phi_{k}=\llbracket \varphi_{k}, \varphi_{k} \rrbracket+\llbracket J \varphi_{k}, J \varphi_{k} \rrbracket=\varphi_{k} \varphi_{k}^{T}+J \varphi_{k} \varphi_{k}^{T} J^{T}, \quad$ where $J=\left[\begin{array}{cc}0 & -I \\ I & 0\end{array}\right]$

Note the following key relations:

$$
\begin{aligned}
\operatorname{real}\left(\left\langle x, f_{k}\right\rangle\right) & =\left\langle\xi, \varphi_{k}\right\rangle \\
\left|\left\langle x, f_{k}\right\rangle\right|^{2} & =\left\langle\Phi_{k} \xi, \xi\right\rangle \\
\operatorname{real}\left(\left\langle x, f_{k}\right\rangle\left\langle f_{k}, y\right\rangle\right) & =\left\langle\Phi_{k} \xi, \eta\right\rangle
\end{aligned}
$$

where $\xi=\mathbf{j}(x)$ and $\eta=\mathbf{j}(y)$. For every $\xi \in \mathbb{R}^{2 n}$ set

$$
R(\xi)=\sum_{k=1}^{m} \llbracket \Phi_{k} \xi, \Phi_{k} \xi \rrbracket
$$

Let $\mathcal{S}^{1,0}$ and $\mathcal{S}^{1,1}$ denote the following spaces of symmetric operators over a Hilbert space $K$ (real or complex)

$$
\begin{aligned}
& \mathcal{S}^{1,0}(K)=\left\{T \in \operatorname{Sym}(K), \operatorname{rank}(T) \leq 1, \lambda_{\max }(T) \geq 0=\lambda_{\min }(T)\right\} \\
& \mathcal{S}^{1,1}(K)=\left\{T \in \operatorname{Sym}(K), \operatorname{rank}(T) \leq 2, \operatorname{Sp}(T)=\left\{\lambda_{\text {max }}, 0, \lambda_{\min }\right\}, \lambda_{\text {max }} \geq 0 \geq \lambda_{\min }\right\}
\end{aligned}
$$

where $\operatorname{Sym}(K)$ denotes the set of symmetric operators (matrices) on $K, S p(T)$ denotes the spectrum (set of eigenvalues) of $T$, and $\lambda_{\max }, \lambda_{\min }$ denote the largest, and smallest eigenvalue of the corresponding operator. Note

$$
\mathcal{S}^{1,0}(K)=\{T=\llbracket x, x \rrbracket, \quad x \in K\}
$$


For the frame $\mathcal{F}=\left\{f_{1}, \ldots, f_{m}\right\}$ we let $\mathcal{A}$ denote the linear operator

$$
\mathcal{A}: \operatorname{Sym}(H) \rightarrow \mathbb{R}^{m} \quad, \quad(\mathcal{A}(T))_{k}=\left\langle T f_{k}, f_{k}\right\rangle=\operatorname{trace}\left(T \llbracket f_{k}, f_{k} \rrbracket\right)
$$

Note the frame condition (11) reads equivalently:

$$
A\|\llbracket x, x \rrbracket\|_{1} \leq\|\mathcal{A}(\llbracket x, x \rrbracket)\|^{2} \leq B\|\llbracket x, x \rrbracket\|_{1}
$$

where $\|T\|_{1}=\sum_{k=1}^{\operatorname{rank}(T)}\left|\lambda_{k}(T)\right|$ denotes the nuclear norm of operator $T$, that is the sum of its singular values, or the sum of magnitudes of its eigenalues when $T$ is symmetric. The upper bound is obviously always true (for an appropriate $B$ ) in the case of finite frames. The lower bound in (1) or (15) is equivalent to the spanning condition $\operatorname{span}(\mathcal{F})=H$. In turn this spanning condition can be restated in terms of a null space condition for $\mathcal{A}$. Specifically let $\operatorname{ker}(\mathcal{A})=\{T \in \operatorname{Sym}(H), \mathcal{A}(T)=0\}$ denote the kernel of the linear operator $\mathcal{A}$. Then $\operatorname{span}(\mathcal{F})=H$ (and therefore $\mathcal{F}$ is frame) if and only if

$$
\operatorname{ker}(\mathcal{A}) \cap \mathcal{S}^{1,0}=\{0\}
$$

Recall the nonlinear map $\alpha$ introduced in (2). We shall consider also the square map $\alpha^{2}$ defined by:

$$
\alpha^{2}: H \rightarrow \mathbb{R}^{m} \quad, \quad\left(\alpha^{2}(x)\right)_{k}=\left|\left\langle x, f_{k}\right\rangle\right|^{2}
$$

Of course $\alpha$ is injective if and only $\alpha^{2}$ is injective.

\section{Stability of Phase Retrievable Frames}

We start by presenting two lemmas regarding the objects we introduced earlier.

Lemma 3.1 Fix a Hilbert space $K$.

(i) As sets, $\mathcal{S}^{1,1}(K)=\mathcal{S}^{1,0}(K)-\mathcal{S}^{1,0}(K)$. Explicitely this means:

$$
\forall T \in \mathcal{S}^{1,1} \exists T_{1}, T_{2} \in \mathcal{S}^{1,0} \quad \text { s.t. } T=T_{1}-T_{2} \quad ; \quad \text { Conversely : } \forall T_{1}, T_{2} \in \mathcal{S}^{1,0}, T_{1}-T_{2} \in \mathcal{S}^{1,1} ;
$$

(ii) For any $T \in \mathcal{S}^{1,1}(K)$ there are $u, v \in K$ so that $T=\llbracket u, v \rrbracket$;

(iii) For any $u, v \in K, \llbracket u, v \rrbracket \in \mathcal{S}^{1,1}(K)$;

(iv) $\mathcal{S}^{1,1}(K)=\{T=\llbracket u, v \rrbracket, u, v \in K\}$.

The proof of this lemma can be found in 7 Lemma 3.7.

Lemma 3.2 The following are equivalent:

(i) The nonlinear map $\alpha$ is injective;

(ii) $\operatorname{ker}(\mathcal{A}) \cap \mathcal{S}^{1,1}=\{0\}$

(iii) There is a constant $a_{0}>0$ so that

$$
\left.\sum_{k=1}^{m}||\left\langle x, f_{k}\right\rangle\right|^{2}-\left.\left|\left\langle y, f_{k}\right\rangle\right|^{2}\right|^{2} \geq a_{0}\left(\|x-y\|^{2}\|x+y\|^{2}+4(\operatorname{imag}(\langle x, y\rangle))^{2}\right)
$$

for any $x, y \in H \in \mathbb{C}^{n}$; 
(iv) There is a constant $a_{0}>0$ so that for all $\xi \in \mathbb{R}^{2 n}, \lambda_{2 n-1}(R(\xi)) \geq a_{0}\|\xi\|^{2}$ (here, $\lambda_{2 n-1}(T)$ denotes the $2 n-1^{\text {th }}$ largest eigenvalue of $T$ );

(v) There is a constant $a_{1}>0$ so that for all $\xi \in \mathbb{R}^{2 n}$,

$$
L(\xi):=R(\xi)+J \llbracket \xi, \xi \rrbracket J^{T}=\sum_{k=1}^{m} \Phi_{k} \xi \xi^{T} \Phi_{k}+J \xi \xi^{T} J^{T} \geq a_{1}\|\xi\|^{2} 1_{\mathbb{R}^{2 n}}
$$

where the inequality is in the sense of quadratic forms;

(vi) For every $\xi \in \mathbb{R}^{2 n}$, dim $\operatorname{ker} R(\xi)=1$;

(vii) For every $\xi \in \mathbb{R}^{2 n}, \operatorname{rank}(R(\xi))=2 n-1$;

(viii) For every $\xi \in \mathbb{R}^{2 n-1}, \operatorname{ker}(R(\xi))=\{c J \xi, c \in \mathbb{R}\}$;

(ix) There is a constant $a_{0}>0$ so that for all $\xi \in \mathbb{R}^{2 n}$,

$$
R(\xi) \geq a_{0}\|\xi\|^{2}\left(1-P_{J \xi}\right)
$$

where $P_{J \xi}=\frac{1}{\|\xi\|^{2}} \llbracket J \xi, J \xi \rrbracket$ is the orthogonal projection onto $J \xi$.

Remark 3.3 Before presenting the proof, note the constants $a_{0}$ at (iii), (iv) and (ix) can be chosen to be equal. Hence the optimal (i.e. the largest) $a_{0}$ is given by

$$
a_{0}=\min _{\|\xi\|=1} \lambda_{2 n-1}(R(\xi))
$$

Additionally, the constant $a_{1}$ at $(v)$ can be chosen as $a_{1}=\min \left(1, a_{0}\right)$.

\section{Proof of Lemma 3.2}

Claims (i),(ii),(iv),(vi)-(ix) are known in literature - see for instance [7 Theorem 2.2 and the bibliographical indications - and Theorem 3.1 of $\left[7\right.$. Claim (v) follows from (ix) by adding $\|\xi\|^{2} P_{J \xi}$ on both sides. Claim (iii) follows from Theorem 3.1 (2) of [7, where we set $u=x-y$ and $v=x+y$ and by remarking $\operatorname{imag}(\langle u, v\rangle)=2 \operatorname{imag}(\langle x, y\rangle)$ and $\operatorname{real}\left(\left\langle u, f_{k}\right\rangle\left\langle f_{k}, v\right\rangle\right)=\operatorname{real}\left(\left|\left\langle x, f_{k}\right\rangle\right|^{2}-\left|\left\langle y, f_{k}\right\rangle\right|^{2}\right)$.

Recall two frames $\mathcal{F}=\left\{f_{1}, \ldots, f_{m}\right\}$ and $\mathcal{G}=\left\{g_{1}, \ldots, g_{m}\right\}$ for the same Hilbert space $H$ are said equivalent if there is an invertible operator $T: H \rightarrow H$ so that $g_{k}=T f_{k}$, for all $1 \leq k \leq m$ (see [3, 32]). The property of being phase retrievable is invariant among equivalent frames, as the following lemma shows.

Lemma 3.4 Assume $\mathcal{F}=\left\{f_{1}, \ldots, f_{m}\right\}$ is a phase retrievable frame for $H$. Then

(i) For any invertible operator $T: H \rightarrow H$ and non-zero scalars $z_{1}, \ldots, z_{m} \in K$, the frame $\mathcal{G}=$ $\left\{g_{1}, \ldots, g_{m}\right\}$ defined by $g_{k}=z_{k} T f_{k}, 1 \leq k \leq m$, is also phase retrievable;

(ii) For any invertible operator $T: H \rightarrow H$, the equivalent frame $\mathcal{G}=\left\{g_{1}, \ldots, g_{m}\right\}$ defined by $g_{k}=T f_{k}$, $1 \leq k \leq m$ is also phase retrievable;

(iii) The canonical dual frame $\tilde{\mathcal{F}}=\left\{\tilde{f}_{1}, \ldots, \tilde{f}_{m}\right\}$ is also phase retrievable, where $\tilde{f}_{k}=S^{-1} f_{k}, 1 \leq k \leq m$; 
(iv) The associated Parseval frame $\mathcal{F}^{\#}=\left\{f_{1}^{\#}, \ldots, f_{m}^{\#}\right\}$ is also phase retrievable, where $f_{k}^{\#}=S^{-1 / 2} f_{k}$, $1 \leq k \leq m$

(v) Any finite set of vectors $\mathcal{G} \subset H$ so that $\mathcal{F} \subset \mathcal{G}$ is a phase retrievable frame;

(vi) If $\mathcal{G} \subset H$ is not a phase retrievable frame then any subset $\mathcal{H} \subset \mathcal{G}$ is also not a phase retrievable frame.

\section{Proof of Lemma 3.4}

(i) Note that each $z_{k} \neq 0$ and hence $\mathcal{G}$ is also frame. Let $\alpha_{\mathcal{G}}: \hat{H} \rightarrow \mathbb{R}^{m}$ be the nonlinear map associated to $\mathcal{G},\left(\alpha_{\mathcal{G}}(x)\right)_{k}=\left|\left\langle x, g_{k}\right\rangle\right|^{2}$. If $x, \in \hat{H}$ are so that $\alpha_{\mathcal{G}}(x)=\alpha_{\mathcal{G}}(y)$ then $\alpha\left(T^{*} x\right)=\alpha\left(T^{*} y\right)$. Since $\mathcal{F}$ is phase retrievable it follows $T^{*} x=T^{*} y$ and hence $x=y$. (Note any operator $R: H \rightarrow H$ lifts to a unique operator $R: \hat{H} \rightarrow \hat{H}$ that is denoted using the same letter).

(ii)-(iv) follows from (i). Claims (v) and (vi) are obvious.

Remark 3.5 While the claim (vi) in previous Lemma is obvious, the following question is not so obvious. Assume $\mathcal{F}$ is a phase retrievable frame in the real case (that is $H=\mathbb{R}^{n}$ ). We know $m \geq 2 n-1$. Assume $m>2 n-1$. Does there always exist a subset $\mathcal{G} \subset \mathcal{F}$ so that $\mathcal{G}$ is a phase retrievable frame? Interestingly the answer to this question is negative. The following example shows this phenomenon (similar example was proposed by [24]).

Example 3.6 Consider $H=\mathbb{R}^{3}$ and $m=6$ where the 6 vectors are:

$$
f_{1}=\left[\begin{array}{l}
1 \\
0 \\
0
\end{array}\right], f_{2}=\left[\begin{array}{l}
0 \\
1 \\
0
\end{array}\right], f_{3}=\left[\begin{array}{l}
0 \\
0 \\
1
\end{array}\right], f_{4}=\left[\begin{array}{l}
1 \\
1 \\
0
\end{array}\right], f_{5}=\left[\begin{array}{l}
1 \\
0 \\
1
\end{array}\right], f_{6}=\left[\begin{array}{l}
0 \\
1 \\
1
\end{array}\right]
$$

The associated rank-1 operators $F_{k}=f_{k} f_{k}^{T}, 1 \leq k \leq 6$, belong to the linear space of symmetric $3 \times 3$ matrices $\operatorname{Sym}\left(\mathbb{R}^{3}\right)$. Note the $\operatorname{Sym}\left(\mathbb{R}^{3}\right)$ is a real vectors space of dimension 6 . The Gram matrix $G^{(2)}$ associated to $\left\{F_{1}, \ldots, F_{6}\right\}$ is a $6 \times 6$ symmetric matrix of entries $G_{k, l}^{(2)}=\left\langle F_{k}, F_{l}\right\rangle=\left|\left\langle f_{k}, f_{l}\right\rangle\right|^{2}$, which are the square of the entries of Gram matrix associated to $\mathcal{F}$. Explicitely $G^{(2)}$ is given by

$$
G^{(2)}=\left[\begin{array}{llllll}
1 & 0 & 0 & 1 & 1 & 0 \\
0 & 1 & 0 & 1 & 0 & 1 \\
0 & 0 & 1 & 0 & 1 & 1 \\
1 & 1 & 0 & 4 & 1 & 1 \\
1 & 0 & 1 & 1 & 4 & 1 \\
0 & 1 & 1 & 1 & 1 & 4
\end{array}\right]
$$

Its determinant is $\operatorname{det}\left(G^{(2)}\right)=8$. Hence $\left\{F_{1}, \ldots, F_{6}\right\}$ is a basis for $\operatorname{Sym}\left(\mathbb{R}^{3}\right)$ and thus $\operatorname{ker}(\mathcal{A})=\{0\}$ which implies $\mathcal{F}$ is a phase retrievable frame. On the other hand consider any subset $\mathcal{G}$ of 5 vectors of $\mathcal{F}$. It is easy to check $\mathcal{G}$ is a frame for $\mathbb{R}^{3}$. Howeverfor each $\mathcal{G}$ there is a subset of 3 elements that is not linearly independent, hence cannot span $\mathbb{R}^{3}$. This fact together with Corollary 2.6 from [8] proves that $\mathcal{G}$ is not phase retrievable. Thus we constructed a frame $\mathcal{F}$ of 6 vectors (which is more than the critical cardinal $2 n-1=5$ ) so that any subset is not phase retrievable.

$5 \mathrm{~mm}$

We are now ready to present the proof of Theorem 1.1

Proof of Theorem 1.1 
Assume $\mathcal{F}$ is a phase retrievable frame. Then equation (19) is satisfied for some $a_{1}>0$. Let $B$ be the upper frame bound for $\mathcal{F}$. Then set:

$$
\rho=\min \left(\frac{1}{\sqrt{m}}, \frac{a_{1}}{4(3 B+2)^{3 / 2}}\right)
$$

We will find a $\rho>0$ so that (19) is satisfied for any set $\mathcal{F}^{\prime}=\left\{f_{1}^{\prime}, \ldots, f_{m}^{\prime}\right\}$ with $\left\|f_{k}-f_{k}^{\prime}\right\|<\rho$. Let $0<A \leq B<\infty$ be the frame bounds of $\mathcal{F}$ and let $L^{\prime}(\xi)$ denote the right hand side in (19) associated to $\mathcal{F}^{\prime}$. We compute

$$
\begin{aligned}
\left|\langle L(\xi) \eta, \eta\rangle-\left\langle L^{\prime}(\xi) \eta, \eta\right\rangle\right| & \leq\left.\sum_{k=1}^{m}||\left\langle\Phi_{k} \xi, \eta\right\rangle\right|^{2}-\left|\left\langle\Phi_{k}^{\prime} \xi, \eta\right\rangle\right|^{2}\left|\leq \sum_{k=1}^{m}\left(\left|\left\langle\Phi_{k} \xi, \eta\right\rangle\right|+\left|\left\langle\Phi_{k}^{\prime} \xi, \eta\right\rangle\right|\right)\right|\left\langle\left(\Phi_{k}-\Phi_{k}^{\prime}\right) \xi, \eta\right\rangle \mid \\
& \leq\left(\sum_{k=1}^{m}\left|\left\langle\Phi_{k} \xi, \eta\right\rangle\right|+\sum_{k=1}^{m}\left|\left\langle\Phi_{k}^{\prime} \xi, \eta\right\rangle\right|\right) \max _{1 \leq k \leq m}\left|\left\langle\left(\Phi_{k}-\Phi_{k}^{\prime}\right) \xi, \eta\right\rangle\right|
\end{aligned}
$$

Fix $\xi \in \mathbb{R}^{2 n}$ with $\|\xi\|=1$. Then

$$
\max _{\|\eta\|=1} \sum_{k=1}^{m}\left|\left\langle\Phi_{k} \xi, \eta\right\rangle\right|=\sum_{k=1}^{m}\left\langle\Phi_{k} \xi, \frac{\xi}{\|\xi\|}\right\rangle \leq B\|\xi\|
$$

Thus for any $\xi, \eta \in \mathbb{R}^{2 n}$,

$$
\sum_{k=1}^{m}\left|\left\langle\Phi_{k} \xi, \eta\right\rangle\right| \leq B\|\xi\|\|\eta\|, \sum_{k=1}^{m}\left|\left\langle\Phi_{k}^{\prime} \xi, \eta\right\rangle\right| \leq B^{\prime}\|\xi\|\|\eta\|
$$

where $B^{\prime}$ is the upper frame bound of $\mathcal{F}^{\prime}$. On the other hand we bound

$$
\left|\left\langle\left(\Phi_{k}-\Phi_{k}^{\prime}\right) \xi, \eta\right\rangle\right| \leq\left\|\Phi_{k}-\Phi_{k}^{\prime}\right\|\|\xi\|\|\eta\|
$$

According to Lemma 3.12 (4) from [7], $\left\|\Phi_{k}-\Phi_{k}^{\prime}\right\|=\left\|F_{k}-F_{k}^{\prime}\right\|$, where $F_{k}=f_{k} f_{k}^{*}$ and $F_{k}^{\prime}=f_{k}^{\prime} f_{k}^{*}$. Note $F_{k}-F_{k}^{\prime} \in \mathcal{S}^{1,1}\left(\mathbb{C}^{n}\right)$ and $F_{k}-F_{k}^{\prime}=\llbracket f_{k}-f_{k}^{\prime}, f_{k}+f_{k}^{\prime} \rrbracket$. Now using Lemma 3.7 (4) from [7, we obtain

$\left\|F_{k}-F_{k}^{\prime}\right\| \leq\left\|F_{k}-F_{k}^{\prime}\right\|_{1}=\sqrt{\left\|f_{k}-f_{k}^{\prime}\right\|^{2}\left\|f_{k}+f_{k}^{\prime}\right\|^{2}+\left(i m a g\left(\left\langle f_{k}-f_{k}^{\prime}, f_{k}+f_{k}^{\prime}\right\rangle\right)\right)^{2}} \leq \sqrt{2}\left\|f_{k}-f_{k}^{\prime}\right\|\left\|f_{k}+f_{k}^{\prime}\right\|$

where $\|T\|_{1}$ is the nuclear norm (the sum of its singular values) of $T$. Next notice $\left\|f_{k}+f_{k}^{\prime}\right\| \leq\left\|f_{k}\right\|+\left\|f_{k}^{\prime}\right\| \leq$ $\sqrt{B}+\sqrt{B^{\prime}} \leq \sqrt{2\left(B+B^{\prime}\right)}$. Putting all the estimates together we obtain:

$$
\left|\langle L(\xi) \eta, \eta\rangle-\left\langle L^{\prime}(\xi) \eta, \eta\right\rangle\right| \leq 2\left(B+B^{\prime}\right)^{3 / 2}\left(\max _{1 \leq k \leq m}\left\|f_{k}-f_{k}^{\prime}\right\|\right)\|\xi\|^{2}\|\eta\|^{2}
$$

Thus

$$
L^{\prime}(\xi) \geq\left(a_{1}-2\left(B+B^{\prime}\right)^{3 / 2} \rho\right)\|\xi\|^{2} 1_{\mathbb{R}^{2 n}}
$$

Finally we obtain an estimate of $B^{\prime}$ in terms of $B, \rho$ and $m$. This estimate can be further refined, but we do not need such a refinment for this proof. Let $\delta_{k}=f_{k}^{\prime}-f_{k}$. Then

$$
\sum_{k=1}^{m}\left|\left\langle x, f_{k}^{\prime}\right\rangle\right|^{2}=\sum_{k=1}^{m}\left|\left\langle x, f_{k}\right\rangle+\left\langle x, \delta_{k}\right\rangle\right|^{2} \leq 2\left(\sum_{k=1}^{m}\left|\left\langle x, f_{k}\right\rangle\right|^{2}+\sum_{k=1}^{m}\left|\left\langle x, \delta_{k}\right\rangle\right|^{2}\right)=2\left(B+m \max _{k}\left\|\delta_{k}\right\|^{2}\right)\|x\|^{2}
$$


Due to (24) we obtain $B^{\prime}=\sup _{\|x\|=1} \sum_{k=1}^{m}\left|\left\langle x, f_{k}^{\prime}\right\rangle\right|^{2} \leq 2(B+1)$. This bound implies that

$$
L^{\prime}(\xi) \geq \frac{a_{1}}{2}\|\xi\|^{2} 1_{\mathbb{R}^{2 n}}
$$

and hence $\mathcal{F}^{\prime}$ is phase retrievable.

\section{Critical case $m=4 n-4$}

This section comments on the recent construction by Bodmann and Hammen [15] of a $4 n-4$ phase retrievable frame in $\mathbb{C}^{n}$. Their construction is as follows. Fix $a \in \mathbb{R} \backslash \pi \mathbb{Q}$, an irrational multiple of $\pi$. The frame set $\mathcal{F}$ is given by a union of two sets, $\mathcal{F}=\mathcal{F}_{1} \cup \mathcal{F}_{2}^{a}$, where $\mathcal{F}_{1}$ constains the following $2 n-3$ vectors:

$$
\mathcal{F}_{1}=\left\{f_{k}^{1}=\left[\begin{array}{lllll}
1 & e^{2 \pi i(k+1) /(2 n-1)} & e^{2 \pi i(k+1) 2 /(2 n-1)} & \cdots & e^{2 \pi i(k+1)(n-1) /(2 n-1)}
\end{array}\right]^{T} \quad, \quad 1 \leq k \leq 2 n-3\right\}
$$

and $\mathcal{F}_{2}^{a}$ contains the following $2 n-1$ vectors:

$$
\mathcal{F}_{2}^{a}=\left\{f_{k}^{2}=\left[\begin{array}{lllll}
1 & z_{k} & z_{k}^{2} & \cdots & z_{k}^{n-1}
\end{array}\right]^{T}, 1 \leq k \leq 2 n-1\right\}
$$

where

$$
z_{k}=\frac{\sin \left(\frac{\pi}{2 n-1}\right)}{\sin (a)} e^{i \frac{k-1}{2 n-1}}-e^{i\left(\frac{\pi}{2 n-1}-\frac{a}{2}\right)} \frac{\sin \left(\frac{\pi}{2 n-1}-\frac{a}{2}\right)}{\sin (a)}
$$

The proof that $\mathcal{F}$ is a phase retrievable frame is based on a result by P. Jamming from [37. Our Theorem 1.1 proves that, in fact, $\mathcal{F}$ remains phase retrievable for a small perturbation. Since $f_{k}^{2}$ depends continuously on $a$, it follows that the set $\mathbb{R} \backslash \pi \mathbb{Q}$ can be replaced by a much larger set of real numbers that includes most of rational multiples of $\pi$. Going through the proof of Theorem 2.3 in [15], and in particular of Lemma 2.2, the only requirement on $a$ is that, any set of $2(n-1)$ complex numbers cannot be simultaneoulsy symmetric with respect to the real line and to a line of angle $a$ passing through the origin. This phenomenon happens for any $n$ when $a$ is an irrational multiple of $\pi$. However, for a fixed $n$, only finitely many values of $a$ may allow such a symmetry. In fact when such a symmetric set of $2(n-1)$ complex numbers exists, $a=\pi \frac{p}{q}$ for some $q \leq 2(n-1)$. Thus the frame set above $\mathcal{F}=\mathcal{F}_{1} \cup \mathcal{F}_{2}^{a}$ remains phase retrievable for all values of $a$ except a finite set of the form $\left\{\pi \frac{p}{q}, 0 \leq p \leq 2 q \leq 4(n-1)\right\}$.

\section{Non Phase-Retrievable Frames}

Consider now the case when $m$ is "small". The conjecture in [11] reads that for $m<4 n-4$ there is no phase retrievable frame. In this section we comment on a partial result supporting this conjecture. The main result of this section is the following

Proposition 5.1 Fix the $n$-dimensional Hilbert space $H$. Denote by $m^{*}(n)$ the critical cardinal $m^{*}(n)=$ $4 n-4$. Assume the following statement holds true for some $m<m^{*}(n)$ :

(O) For any frame set $\mathcal{F}=\left\{f_{1}, \ldots, f_{m}\right\} \subset H$ that is not a phase retrievable frame for $H$ there exists a $\rho>0$ so that any other set $\mathcal{F}^{\prime}=\left\{f_{1}^{\prime}, \ldots, f_{m}^{\prime}\right\}$ with $\left\|f_{k}-f_{k}^{\prime}\right\|<\rho, 1 \leq k \leq m$, is also not phase retrievable.

Then any subset $\mathcal{G}=\left\{g_{1}, \ldots, g_{m}\right\} \subset H$ of $m$ vectors in $H$ is not a phase retrievable frame. 
Remark 5.2 Before presenting its proof, we make the following remark. While not proving the fulll conjecture, this result reduces the proof of the $4 n-4$ conjecture to a stability result for non phase retrievable frames. Additionally the result holds true even if the critical cardinal is not $4 n-4$. However the author recognizes this is just a partial result that does not prove the full conjecture.

Remark 5.3 Note the set $\mathcal{F}$ is supposed to be frame. If $(O)$ holds for any set of $m$ vectors of $H$, one can use the trivial set $\{0,0, \ldots, 0\}$ of $m$ vectors. Then if $(O)$ holds for this special set, then any set of $m$ vectors whose norms are less than some $\rho>0$ is non phase retrievable frame. By scaling we obtin immediately that any $m$-set of vectors is not phase retrievable frame.

\section{Proof of Proposition 5.1}

First note that if $m<2 n$ any set $\mathcal{F}$ of $m$ elements cannot be a phase retrievable frame (conform [28]). Hence we can assume $m \geq 2 n$. Let $H^{m}=H \times H \times \cdots \times H$ denote the $m$-product space endowed with the topology induced by the norm

$$
\|\mathbf{x}\|_{H^{m}}=\max _{1 \leq k \leq m}\left\|x^{k}\right\| \quad, \quad \mathbf{x}=\left(x^{1}, \ldots, x^{m}\right) \in H^{m}
$$

Note that $H^{m}$ is homeomorphic with $\mathbb{C}^{n m}$ endowed with the usual Euclidian norm. Let $F_{n}^{m}$ denote the set of frames for $H$ with $m$ elements. $F_{n}^{m}$ is an open set in $H^{m}$ since each frame set is stable under small perturbations: for instance this can be seen using an estimate similar to equation (25) that bounds below $\sum_{k=1}^{m}\left|\left\langle x, f_{k}^{\prime}\right\rangle\right|^{2}$ :

$$
\begin{aligned}
\sum_{k=1}^{m}\left|\left\langle x, f_{k}^{\prime}\right\rangle\right|^{2} & \geq \sum_{k=1}^{m}\left|\left\langle x, f_{k}\right\rangle\right|^{2}-\left(2 \sqrt{m}\left(\sum_{k=1}^{m}\left|\left\langle x, f_{k}\right\rangle\right|^{2}\right)^{1 / 2}\left(\max _{k}\left\|f_{k}-f_{k}^{\prime}\right\|\right)\|x\|+m\left(\max _{k}\left\|f_{k}-f_{k}^{\prime}\right\|\right)^{2}\|x\|^{2}\right) \\
& \geq\left(A-2 \sqrt{m B} \rho-m \rho^{2}\right)\|x\|^{2}
\end{aligned}
$$

Thus for some sufiiciently small $\rho, A-2 \sqrt{m B} \rho-m \rho^{2}>0$ and $\left\{f_{1}^{\prime}, \ldots, f_{m}^{\prime}\right\}$ is also frame when $\left\|f_{k}-f_{k}^{\prime}\right\|<\rho$, $1 \leq k \leq m$.

Assume the hypothesis $(\mathrm{O})$ holds true. Let $N_{n}^{m}$ denote the set of non phase-retrievable frames of $m$ vectors in $H$. Thus $N_{n}^{m} \subset F_{n}^{m} \subset H^{m}$ is an open set in $H$ by hypothesis (O).

On the other hand the complement $\Gamma_{n}^{m}:=F_{n}^{m} \backslash N_{n}^{m}$ represents the set of phase retrievable frames. Theorem 1.1 shows $\Gamma_{n}^{m}$ is open in $H^{m}$.

Now let us show the set of frames $F_{n}^{m}$ is connected in $H^{m}$. Firstly two equivalent frames are connected by path as shown e.g. in [3]. We will show that any two frames of $m$ elements for the $n$ dimensional Hilbert space $H$ can be connected by a continuous path (in fact two segments of line), when $m \geq 2 n$. Let $\mathcal{F}_{1}=\left\{f_{1}^{1}, \ldots, f_{m}^{1}\right\}$ and $\mathcal{F}_{2}=\left\{f_{1}^{2}, \ldots, f_{m}^{2}\right\}$ be two $m$-frames. Let $I=\left\{k_{1}, \ldots, k_{n}\right\}$ be the $n$-set so that $\mathcal{F}_{1}[I]=\left\{f_{k_{1}}^{1}, \ldots, f_{k_{n}}^{1}\right\}$ is a linearly independent subset of $\mathcal{F}_{1}$. Let $J=\left\{j_{1}, \ldots, j_{m-n}\right\}$ be a $m-n$-set so that $\mathcal{F}_{2}[J]=\left\{f_{j}^{2}, j \in J\right\}$ is a frame for $H$. Let $\gamma: I^{c} \rightarrow J$ and $\delta: J^{c} \rightarrow I$ be two bijective maps where $I^{c}=\{1 \leq k \leq m\} \backslash I$ and $J^{c}=\{1 \leq j \leq m\} \backslash J$ are the complement sets of $I$ and $J$ respectively. We build a piecewise linear path $\beta:[-1,1] \rightarrow H^{m}$ connecting $\mathcal{F}_{1}$ to $\mathcal{F}_{2}$ as follows: For $-1 \leq t \leq 0$,

$$
(\beta(t))_{k}=\left\{\begin{array}{cll}
f_{k}^{1} & \text { if } & k \in I \\
-t f_{k}^{1}+(t+1) f_{\gamma(k)}^{2} & \text { if } & k \in I^{c}
\end{array}\right.
$$

For $0 \leq t \leq 1$,

$$
(\beta(t))_{k}=\left\{\begin{array}{ccc}
f_{j}^{2} & \text { if } & j \in J \\
t f_{\delta(j)}^{1}+(1-t) f_{j}^{2} & \text { if } & j \in J^{c}
\end{array}\right.
$$


One can easilty check that $\beta(t)$ is a frame for each $-1 \leq t \leq 1$, and $\beta(-1)=\mathcal{F}_{1}, \beta(1)=\mathcal{F}_{2}$. This proves the set of frames $F_{n}^{m}$ is path connected, hence connected.

We obtained that the connected set $F_{n}^{m}$ can be partitioned into two opens sets $\Gamma_{n}^{m}$ and $N_{n}^{m}$. It follows that one of the two sets must be the empty set. However we can always construct a non phase retrievable frame, for instance $\mathcal{F}=\left\{e_{1}, \ldots, e_{n}, e_{n}, \ldots, e_{n}\right\}$ where $\left\{e_{1}, \ldots, e_{n}\right\}$ is a basis of $H$ and the vector $e_{n}$ is repeated a total of $m-n+1$ times. This shows $\Gamma_{n}^{m}$ must be empty. Thus any set $\mathcal{G} \subset H$ of $m$ vectors cannot be a phase retrievable frame.

\section{ACKNOWLEDGMENTS}

The author has been partially supported by the National Science Foundation under NSF DMS-1109498 grant.

\section{References}

[1] B. Alexeev, A. S. Bandeira, M. Fickus, D. G. Mixon, Phase Retrieval with Polarization, online arXiv:1210.7752v1 [cs.IT] 29 Oct 2012.

[2] D. M. Appleby, Symmetric informationally complete-positive operator valued measures and the extended Clifford group, J. Math. Phys. 46 (2005), no. 5, 052107, 29.

[3] R. Balan, Equivalence relations and distances between Hilbert frames, Proc. Amer. Math. Soc. 127 (1999), no. $8,2353-2366$.

[4] R. Balan, A Nonlinear Reconstruction Algorithm from Absolute Value of Frame Coefficients for Low Redundancy Frames, Proceedings of SampTA Conference, Marseille, France May 2009.

[5] R. Balan, On Signal Reconstruction from Its Spectrogram, Proceedings of the CISS Conference, Princeton NJ, May 2010.

[6] R. Balan, Reconstruction of Signals from Magnitudes of Redundant Representations, online arXiv:1207.1134v1 [math.FA] 4 July 2012.

[7] R. Balan, Reconstruction of Signals from Magnitudes of Redundant Representations: The Complex Case, online arXiv:1304.1839v1 [math.FA] 6 April 2013.

[8] R. Balan, P. Casazza, D. Edidin, On signal reconstruction without phase, Appl.Comput.Harmon.Anal. 20 (2006), 345-356.

[9] R. Balan, P. Casazza, D. Edidin, Equivalence of Reconstruction from the Absolute Value of the Frame Coefficients to a Sparse Representation Problem, IEEE Signal.Proc.Letters, 14 (5) (2007), 341-343.

[10] R. Balan, B. Bodmann, P. Casazza, D. Edidin, Painless reconstruction from Magnitudes of Frame Coefficients, J.Fourier Anal.Applic., 15 (4) (2009), 488-501.

[11] A.S. Bandeira, J. Cahill, D.G. Mixon, A.A. Nelson, Saving phase: Injectivity and stability for phase retrieval, online arXiv:1302.4618v2 [math.FA] 14 Mar 2013. 
[12] S. Bandyopadhyay, P. O. Boykin, V. Roychowdhury, and F. Vatan, A new proof for the existence of mutually unbiased bases, Algorithmica 34 (2002), no. 4, 512-528, Quantum computation and quantum cryptography.

[13] B. G. Bodmann, Optimal linear transmission by loss-insensitive packet encoding, Appl. Comput. Harmon. Anal. 22 (2007), 274-285.

[14] B. G. Bodmann and V. I. Paulsen, Frames, graphs and erasures, Linear Algebra Appl. 404 (2005), $118-146$.

[15] B. G. Bodmann, N. Hammen, Stable Phase Retrieval with Low-Redundancy Frames, online arXiv submission: $1302.5487 \mathrm{v} 1$

[16] P. O. Boykin, M. Sitharam, P. H. Tiep, and P. Wocjan Mutually unbiased bases and orthogonal decompositions of Lie algebras, E-print: arXiv.org/quant-ph/0506089, to appear in Quantum Information and Computation, 2007.

[17] A. R. Calderbank, P. J. Cameron, W. M. Kantor, and J. J. Seidel, $Z_{4}$-Kerdock codes, orthogonal spreads, and extremal Euclidean line-sets, Proc. London Math. Soc. (3) 75 (1997), no. 2, 436-480.

[18] E. Candés, T. Strohmer, V. Voroninski, PhaseLift: Exact and Stable Signal Recovery from Magnitude Measurements via Convex Programming, to appear in Communications in Pure and Applied Mathematics (2011)

[19] E. Candés, Y. Eldar, T. Strohmer, V. Voroninski, Phase Retrieval via Matrix Completion Problem, preprint 2011

[20] P. Casazza, The art of frame theory, Taiwanese J. Math., (2) 4 (2000), 129-202.

[21] P. G. Casazza and M. Fickus, Fourier transforms of finite chirps, EURASIP J. Appl. Signal Process. (2006), no. Frames and overcomplete representations in signal processing, communications, and information theory, Art. ID 70204, 1-7.

[22] P. Casazza and J. Kovačević, Equal-norm tight frames with erasures, Adv. Comp. Math. 18 (2003), $387-430$.

[23] P. G. Casazza and G. Kutyniok, Frames of subspaces, Wavelets, frames and operator theory, Contemp. Math., vol. 345, Amer. Math. Soc., Providence, RI, 2004, pp. 87-113.

[24] J. Cahill, personal communication, October 2012.

[25] P. J. Cameron and J. J. Seidel, Quadratic forms over GF(2), Indag. Math. 35 (1973), 1-8.

[26] P. Delsarte, J. M. Goethals, and J. J. Seidel, Spherical codes and designs, Geometriae Dedicata 6 (1977), no. $3,363-388$.

[27] Y. C. Eldar, S. Mendelson, Phase retrieval: Stability and recovery guarantees, Available online: arXiv:1211.0872

[28] J. Finkelstein, Pure-state informationally complete and "really" complete measurements, Phys. Rev. A 70 (2004), no. 5, doi:10.1103/PhysRevA.70.052107 
[29] C. Godsil and A. Roy, Equiangular lines, mutually unbiased bases and spin models, 2005, E-print: arxiv.org/quant-ph/0511004.

[30] V. K. Goyal, J. Kovačević, and J. A. Kelner, Quantized frame expansions with erasures, Appl. Comp. Harm. Anal. 10 (2001), 203-233.

[31] M. H. Hayes, J. S. Lim, and A. V. Oppenheim, Signal Reconstruction from Phase and Magnitude, IEEE Trans. ASSP 28, no.6 (1980), 672-680.

[32] D. Han, D. R. Larson, Frames, Bases and Group Representations, Mem. Am. Math. Soc. 147(2000), no. 697,94 pages.

[33] T. Heinosaari, L. Mazzarella, M. M. Wolf, Quantum Tomography under Prior Information, arXiv:1109.5478v1 [quant-ph], 26 Sept 2011.

[34] S. G. Hoggar, t-designs in projective spaces, Europ. J. Comb. 3 (1982), 233-254.

[35] S. D. Howard, A. R. Calderbank, and W. Moran, The finite Heisenberg-Weyl groups in radar and communications, EURASIP J. Appl. Signal Process. (2006), no. Frames and overcomplete representations in signal processing, communications, and information theory, Art. ID 85685, 1-12.

[36] R. Holmes and V. I. Paulsen, Optimal frames for erasures, Lin. Alg. Appl. 377 (2004), 31-51.

[37] P. Jaming, Uniqueness results for the phase retrieval problem of fractional Fourier transforms of variable order, preprint, arXiv:1009.3418,

[38] S. M. Kay, Fundamentals of Statistical Signal Processing. I. Estimation Theory, Prentice Hall PTR, 18th Printing, 2010.

[39] J. Kovačević, P. L. Dragotti, and V. K. Goyal, Filter bank frame expansions with erasures, IEEE Trans. Inform. Theory 48 (2002), 1439-1450.

[40] A. Klappenecker and M. Rötteler, Mutually unbiased bases are complex projective 2-designs, Proc. Int. Symp. on Inf. Theory, IEEE, 2005, pp. 1740-1744.

[41] R. G. Lane, W. R. Freight, and R. H. T. Bates, Direct Phase Retrieval, IEEE Trans. ASSP 35, no.4 (1987), 520-526.

[42] P. W. H. Lemmens and J. J. Seidel, Equiangular lines, J. Algebra 24 (1973), 494-512.

[43] Yu. I. Lyubich, On tight projective designs, E-print: www.arXiv.org/math/0703526, 2007.

[44] D. Mondragon, V. Voroninski, Determination of all pure quantum states from a minimal number of observables, online arXiv:1306.1214v1 [math-ph] 5 June 2013.

[45] R. J. Milgram, Immersing Projective Spaces, Annals of Mathematics, vol. 85, no. 3 (1967), 473-482.

[46] H. Nawab, T. F. Quatieri, and J. S. Lim, Signal Reconstruction from the Short-Time Fourier Transform Magnitude, in Proceedings of ICASSP 1984.

[47] A. Neumaier, Combinatorial congurations in terms of distances, Memorandum 81-09 (Wiskunde), Eindhoven Univ.Technol., (1981). 
[48] T. Strohmer and R. Heath, Grassmannian frames with applications to coding and communcations, Appl. Comp. Harm. Anal. 14 (2003), 257-275.

[49] J. H. van Lint and J. J. Seidel, Equilateral point sets in elliptic geometry, Indag. Math. 28 (1966), $335-348$.

[50] R. Vale and S. Waldron, Tight frames and their symmetries, Constr. Approx. 21 (2005), no. 1, 83-112.

[51] I. Waldspurger, A. dAspremont, S. Mallat, Phase recovery, MaxCut and complex semidefinite programming, Available online: arXiv:1206.0102

[52] Y. Wang, Random matrices and erasure robust frames, preprint.

[53] Y. Wang, preliminary report.

[54] W. K. Wootters and B. D. Fields, Optimal state-determination by mutually unbiased measurements, Ann. Physics 191 (1989), no. 2, 363-381. 